\title{
A Computational Model of Collective Sensemaking: Differential Effects of Communication Network Structure on Collective Sensemaking Abilities
}

Paul R. Smart (ps02v@ecs.soton.ac.uk)

School of Electronics and Computer Science, University of Southampton, Southampton, SO17 1BJ, UK

\section{INTRODUCTION}

Collective sensemaking is a form of socially-distributed cognition (see Hutchins, 1995) in which multiple agents attempt to interpret (make sense of) specific bodies of environmental information. In order to optimize performance at the collective level, agents often need to share information about the results of their own processing activity, and this raises questions about how the structure of communication networks affects collective sensemaking abilities. In the current study, we used a computational model of collective sensemaking in which individual agents were implemented as constraint satisfaction networks (CSNs) (see Smart and Shadbolt, 2012). We then investigated how the cognitive responses of agents were affected by different kinds of communication network structure.

\section{METHOD}

A multi-agent computational model was used in which agents were implemented as CSNs. All agents had the same architecture, which consisted of 6 cognitive units connected together via inhibitory and excitatory connections (see Figure 1). The computational model was based on a previous model developed by Schultz and Lepper (1996). They used CSNs to explore the dynamics of cognitive dissonance reduction.

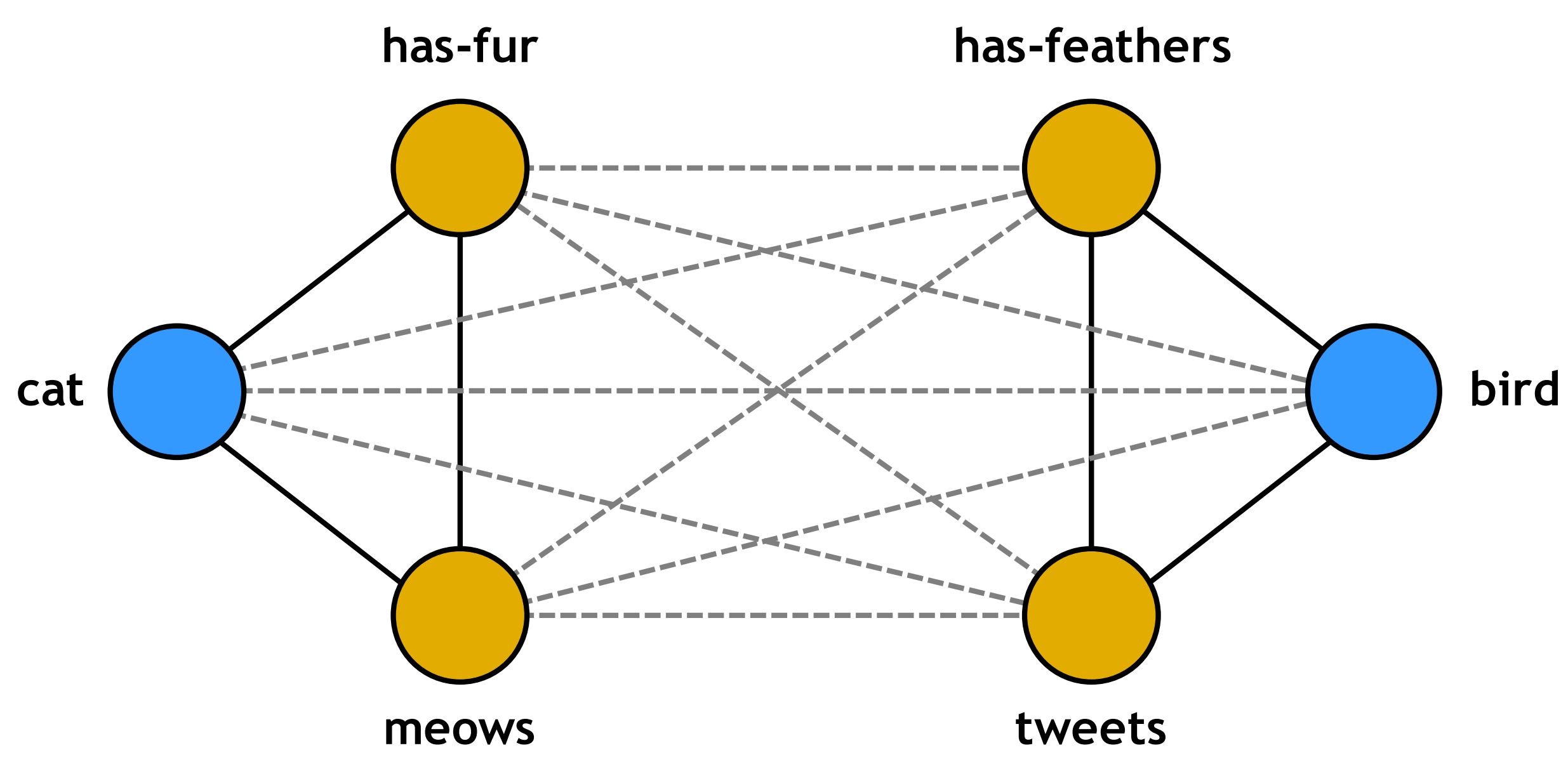

Figure 1. Organization of cognitive units in a single agent.

The cognitive units were connected together in such a way as to yield two kinds of interpretive response to environmental information. On the one hand, agents could interpret environmental information as indicating the presence of a cat, and, on the other hand, they could interpret environmental information as indicating the presence of a bird. Across the course of each simulation, one of these cognitive responses tended to predominate due to the pattern of excitatory and inhibitory links between cognitive units.

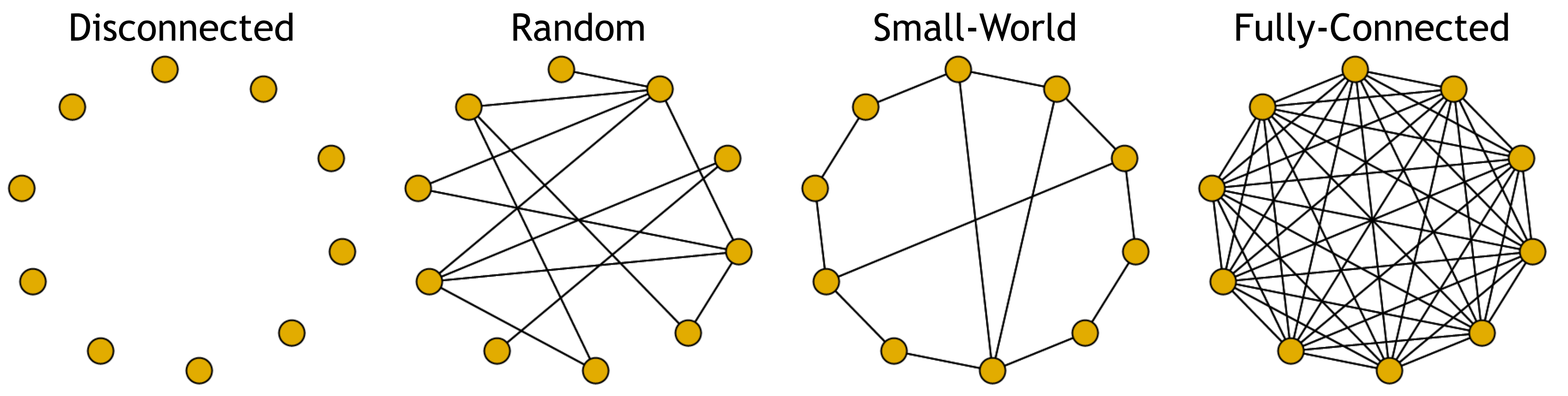

Figure 2. Examples of the network structures used in the study.

In each simulation, 10 agents were organized into one of four types of communication network structure (see Figure 2). These structures constrained the way in which agents shared information about their beliefs.

\begin{tabular}{|l|l|l|l|l|l|l|}
\hline & has-fur & meows & cat & feathers & tweets & bird \\
\hline Ambiguous & 0.1 & 0.1 & 0.0 & 0.2 & 0.2 & 0.2 \\
\hline Unambiguous & 0.5 & 0.5 & 0.0 & 0.0 & 0.0 & 0.0 \\
\hline
\end{tabular}

Table 1. Activation vectors used in the study.

Once the network structure had been created, the activation levels of cognitive units within each agent were initialized using one of two types of activation vector (see Table 1 ). At the start of each simulation, 4 agents were selected at random and were initialized with the 'Unambiguous' activation vector; the remainder of the agents were initialized with the 'Ambiguous' activation vector.

A total of 50 simulations were run in each of the four network structure conditions. Each simulation consisted of 20 processing cycles. At the end of each simulation, the activation of the 'Cat' and 'Bird' cognitive units was recorded for subsequent analysis.

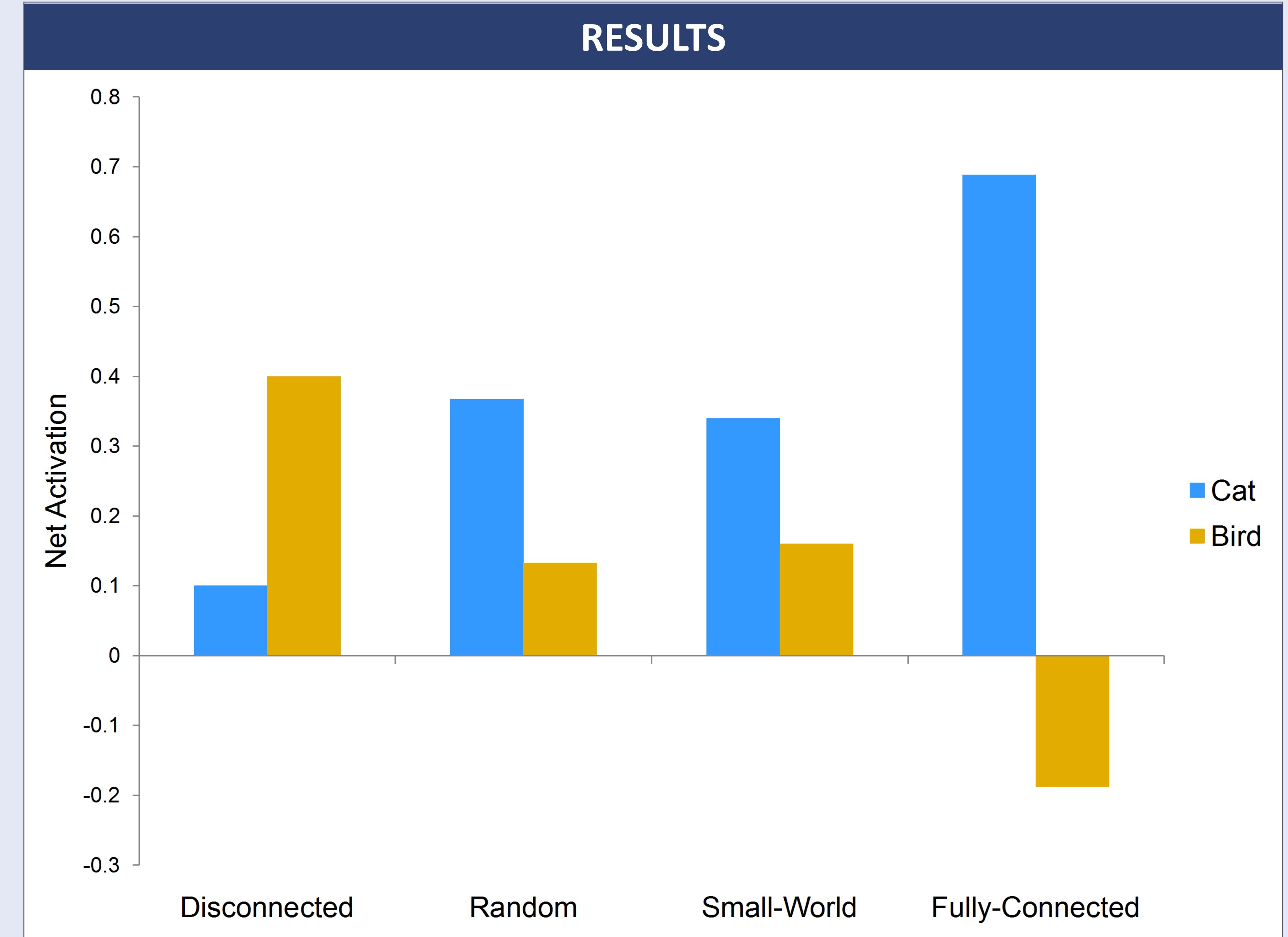

Figure 3. Mean responses of 'Cat' and 'Bird' cognitive units in each of the four network structure conditions.

The results are shown in Figure 3. ANOVA revealed a significant main effect of Cognitive Unit (i.e. activation of the 'Cat' and 'Bird' cognitive units) and a significant two-way interaction between the Network Structure and Cognitive Unit factors. There was no significant main effect of Network Structure (using a conservative alpha criterion of 0.01 ). Post hoc comparisons using Tukey's HSD test were performed at each level of the Cognitive Unit factor. These analyses revealed that cognitive responses in the random and small-world network conditions were not significantly different from each other for either the 'Bird' or 'Cat' cognitive units. The activation level of the 'Cat' cognitive unit was higher in both the random and small-world network conditions as compared to the disconnected network condition, and the activation of the 'Bird' cognitive unit was lower in the random and small-world network conditions as compared to the disconnected network condition. Activation of the 'Cat' cognitive unit was higher in the fully-connected network as compared to all other networks, and activation of the 'Bird' unit was lower in the fully-connected network as compared to all other networks. Post hoc comparisons of the cognitive responses for each of the network structures revealed significant differences between the activation of 'Cat' and 'Bird' cognitive units for all network conditions. Activation of 'Cat' cognitive units was higher than 'Bird' cognitive units for all networks, with the exception of disconnected networks.

\section{CONCLUSION}

The results of this study suggest that collective sensemaking is influenced by network structure under certain informational conditions. In all of the conditions in which agents were allowed to communicate information, a particular kind of cognitive response emerged in which cat-related beliefs predominated. This differed from the situation in which agents were not allowed to communicate (i.e. the disconnected network condition). The cognitive responses of agents that were organized into random and smallworld network topologies were very similar; however, they were less extreme than those of agents organized into fully-connected network topologies. Further work in this area may help to reveal how the structure of inter-agent communication affects the dynamics of collective cognitive processing.

\section{REFERENCES}

Hutchins, E. (1995). Cognition in the Wild. Cambridge, Massachusetts, USA: MIT Press.

Schultz, T. R., \& Lepper, M. R. (1996). Cognitive dissonance reduction as constraint satisfaction. Psychological Review, 103(2), 219-240.

Smart, P. R., \& Shadbolt, N. R. (2012). Modelling the dynamics of team sensemaking: A constraint satisfaction approach. In Knowledge Systems for Coalition Operations (KSCO'12). Pensacola, Florida, USA. 